\title{
Topological regulation of activation barriers on fractal substrates
}

\author{
Raffaella Burioni \\ Dipartimento di Fisica e Scienza della Terra, and INFN, \\ Gruppo Collegato di Parma, Università di Parma, \\ Parco Area delle Scienze 7/A, I-423100 Parma, Italy. \\ Federico Corberi \\ Dipartimento di Fisica "E. R. Caianiello", and INFN, \\ Gruppo Collegato di Salerno, and CNISM, Unità di Salerno, \\ Università di Salerno, via Ponte don Melillo, 84084 Fisciano (SA), Italy. \\ Alessandro Vezzani \\ Centro S3, CNR-Istituto di Nanoscienze, Via Campi 213A, \\ 41125 Modena Italy, and Dipartimento di Fisica Scienza della Terra, \\ Università di Parma, Parco Area delle Scienze 7/A, I-43100 Parma, Italy.
}

\begin{abstract}
We study phase-ordering dynamics of a ferromagnetic system with a scalar order-parameter on fractal graphs. We propose a scaling approach, inspired by renormalization group ideas, where a crossover between distinct dynamical behaviors is induced by the presence of a length $\lambda$ associated to the topological properties of the graph. The transition between the early and the asymptotic stage is observed when the typical size $L(t)$ of the growing ordered domains reaches the crossover length $\lambda$. We consider two classes of inhomogeneous substrates, with different activated processes, where the effects of the free energy barriers can be analytically controlled during the evolution. On finitely ramified graphs the free energy barriers encountered by domains walls grow logarithmically with $L(t)$ while they increase as a power-law on all the other structures. This produces different asymptotic growth laws (power-laws vs logarithmic) and different dependence of the crossover length $\lambda$ on the model parameters. Our theoretical picture agrees very well with extensive numerical simulations.
\end{abstract}

\section{INTRODUCTION}

The slow relaxation of glasses, spin-glasses and phase-separating systems is a subject of paramount importance in non-equilibrium statistical mechanics. In the case of homogeneous ferromagnets, i.e non-disordered magnetic systems defined on homogenous substrates such as lattices, the basic features of the dynamics after a quench below the critical temperature are well understood in terms of the domain growth mechanism [1]. The unbounded growth of the size of ordered domains with nearly equilibrium composition involve a scaling symmetry due to the presence of a dominant length scale, which is manifested in the lack of time-translational invariance and aging. Although this simple paradigm of slow relaxation is expected to encompass a broad variety of situations, its applicability to more complex systems, such as spin-glasses, where both homogeneity and ferromagnetism are spoiled, remains a debated issue [2]. An intermediate class of systems is that of non-homogeneous ferromagnetic materials [3]. In these systems space homogeneity is wrecked by spacial modulations of some relevant parameter which, in order to maintain the system ferromagnetic, must coexist with the low-temperature order. In this case phase-ordering kinetics is preserved, although interesting new features may arise. The agent whereby homogeneity is spoiled may be random, like random fields, random bonds, or dilution, or deterministic, as in the case of models defined on deterministic graphs. The kinetics of these systems have been an active area of research for quite some time now. An unifying feature is the ubiquitous appearance of energetic barriers slowing down the motion of domains boundaries. This happens because, due to space inhomogeneity, interfaces are pinned in particular positions, and this may have dramatic consequences on the growth law and on the properties of correlation functions [4 7]. Despite many experimental and theoretical studies [3], a number of issues are still open. In particular, the conditions under which inhomogeneities may radically modify the asymptotic dynamics are not a priori known [8]. Indeed there are cases where disorder merely change the time units, like the one-dimensional Ising model with random bond [9], and others, like the same model but with disorder in the form of a random-field 10], where profound qualitative modifications occur. For this second class of systems the nature of the dynamical scaling symmetry and of the asymptotic growth law is not yet fully characterized. Related to that, the conjecture of a superuniversal behavior, namely the idea that scaling functions are robust with respect to the presence on inhomogeneities which do not to change the low temperature properties of the system [11], has been proposed.

Recently, in the context of disordered ferromagnets, the observed behavior has been interpreted [12] in terms of a picture inspired by renormalization group ideas where disorder introduces, next to the domains size, another characteristic length which gives rise to a crossover pattern. In this paper we apply similar ideas to the realm of 
ferromagnets defined on non-homogeneous physical 13] fractal graphs, which turn out to be a simple but paradigmatic case where energy barriers thoroughly modify the asymptotic behavior of phase ordering dynamics. Indeed, at variance with the case of disordered systems, the nature of the energetic barriers and their scaling properties can be reasonably well understood and they can be related to the topological inhomogeneity. This allows one to make precise predictions on the growth laws, on the scaling properties and on the crossover phenomena. These predictions conform quite well to the outcome of numerical simulations of the dynamics of the Ising model on graphs. The main result of this paper is the existence of a crossover from an early power-law behavior to a slower asymptotic growth. The latter may be power-law (with a smaller, temperature dependent exponent) or logarithmic according to the structure of the barriers, which in turn depend on the topology of the graph. In particular, we conjecture power-laws to be associated to graphs which sustain ferromagnetic order only at zero temperature, while logarithms are expected if an ordered phase exists. The scaling functions of correlation functions are sensitive to the crossover.

This paper is organized as follows. In Sec. II. we provide an overview of domain growth laws and of the scaling framework proposed in [12] for disordered systems. In Sec. [II] we specify and adapt the scenario to the case of ferromagnets on graphs, providing also predictions for several quantities such as crossover lengths and exponents. In Sec. IV] we present numerical results for the phase-ordering of the Ising model on three model fractal graphs, the Sierpinski carpet (SC), the Sierpinski gasket (SG), and the T-fractal (TF). For the first structure a low-temperature ordered phase exists, whereas it is not sustained in the other two. We will argue this fact to represent a basic difference for the dynamical evolution. The numerical results are interpreted using the scaling ideas of Secs. IIIII and agree quite nicely with the predictions obtained there. In Sec. V] we conclude the paper with a summary and a discussion on the generality of our results.

\section{GROWTH LAWS AND DYNAMICAL SCALINGS}

Domain growth kinetics is characterized by an ever increasing typical domain size $L(t)$ after the quench of a system to a final temperature $T$ in the ordered phase. When $L(t)$ becomes the dominant length in the problem, dynamical scaling is observed, meaning that all other lengths can be measured in units of $L(t)$. Such property is reflected for instance in the two-point/two-time order parameter correlation function $\mathcal{G}\left(r ; t, t_{w}\right)$, where $r$ is the distance between the two points and $t>t_{w}$, which in homogeneous systems scales as [14]

$$
\mathcal{G}\left(r ; t, t_{w}\right)=\widehat{\mathcal{G}}\left[r / L(t), L\left(t_{w}\right) / L(t)\right]
$$

$\widehat{\mathcal{G}}$ being a scaling function.

Generally speaking, the growth law and other properties such as $\widehat{\mathcal{G}}$ may depend on several factors, e.g. temperature, conservation laws, dimensionality, order parameter symmetry, disorder and topology of the substrate. In the simplest cases, as in homogeneous magnets with non-conserved dynamics, the kinetics proceeds without activation events. This means that no free energy barriers are encountered in the evolution and, consequently, phase-ordering is observed down to temperature $T=0$. In this case the typical domains size increases as $L(t) \sim t^{1 / 2}$. On the other hand, the kinetics of other coarsening systems require thermal activation. The simplest example are perhaps homogeneous magnets with conserved dynamics. In this case energy barriers arise as due to the microscopic evaporation-condensation mechanism underlying the evolution [15]. In general, barriers can have different origin and scaling properties. In non-homogeneous systems, like the disordered ones or those embedded on a non-homogeneous substrate, they are typically due to the pinning of interfaces in certain preferred positions. In a wide class of disordered systems, such as the Ising model with random fields [10, 12, 16, 17], random bonds [18], or random dilution [7], this pinning effect slows down asymptotically the growth law to a logarithmic form

$$
L(t) \propto[\ln (t / \tau)]^{1 / \psi},
$$

where $\tau$ and $\psi$ are model-dependent quantities. With an Arrhenius form

$$
t \propto e^{\mathcal{E} /\left(k_{B} T\right)}
$$

for the time needed to escape a barrier with activation free energy $\mathcal{E}$, the problem of establishing the growth law is directly related to the one of determining the typical height $\mathcal{E}$ of barriers at a certain stage of the evolution. Indeed, inverting the relation (3) one concludes that the typical activation increases with the domains size as a power-law:

$$
\mathcal{E}(L) \propto L^{\psi}
$$

Eq. (4) is not the only possible situation, since a different algebraic growth law

$$
L(t) \propto t^{1 / \zeta},
$$


with $\zeta$ a temperature dependent exponent, is observed asymptotically in systems defined on a class of fractal structures [19, 20] and pre-asymptotically in random field [12] and random bond ferromagnets [18, 21]. Plugging Eq. (5) in Eq. (3) one arrives at

$$
\mathcal{E}(L) \propto\left[\ln (L)^{\zeta}\right],
$$

for the scaling of the barriers with the domains size. A classification of phase-ordering systems according to the possible growth laws is made in [8]. In general, however, a complete characterization of $L(t)$ in the many varied instances of coarsening systems is lacking. Besides that, understanding the behavior of the scaling functions such as $\widehat{\mathcal{G}}$ in Eq. (1) is also an open problem.

In Ref. [12], it was proposed to unify the wide variety of behaviors observed in domain growth into a generalized scaling framework. The basic idea, originally conceived for disordered systems, is that the agent responsible for inhomogeneity introduces an extra characteristic length $\lambda$. In the case of the Ising model with non-conserved dynamics defined on a inhomogeneous graph considered in this paper (see section III), the only parameters of the model are the ferromagnetic coupling constant $J$ and $T$, which enter in the combination $\epsilon=J / T$. Then it must be $\lambda=\lambda(\epsilon)$. The presence of $\lambda$ introduces a crossover phenomenon between two different dynamical behaviors when $L(t)$ crosses $\lambda$. For the domains size this is assumed to be described by

$$
L(t ; \epsilon)=t^{1 / z} \widehat{L}\left(\epsilon / t^{\phi}\right),
$$

where $z$ and $\phi$ are a growth and a crossover exponent, with a scaling function behaving as

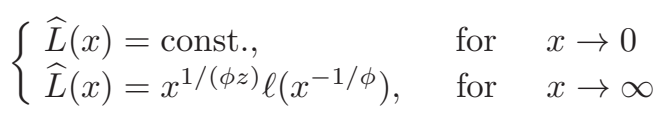

where $x=\epsilon / t^{\phi}$. In systems where disorder plays the role of an irrelevant parameter $\phi$ is positive, inhomogenities only affects the dynamics at early times and $L(t)$ crosses over to the pure system behavior $L(t) \sim t^{1 / z}$ at large times [12]. This usually happens if there is an upper limit for the height of energetic barriers. On the other hand, when barriers of any size can be encountered, as in the fractal models considered in this paper and others, $\phi$ is negative and Eq. (8) describes the crossover from the early stage power law

$$
L(t) \sim t^{1 / z},
$$

with a temperature independent growth exponent $z$, to the asymptotic form $L(t)=\lambda(\epsilon) \ell\left(t / \epsilon^{1 / \phi}\right)$ at the crossover length $\lambda=\epsilon^{1 /(\phi z)}$. The functions $\lambda(\epsilon)$ and $\ell\left(t / \epsilon^{1 / \phi}\right)$ are in general hard to be determined, both analytically and numerically. However, some prediction can be made in the case of coarsening on deterministic fractal structures, as will be discussed in Sec. III.

\section{A. Autocorrelation function}

In order to discuss the effect of inhomogeneities on the form of the correlation functions let us consider as a paradigm the autocorrelation function $C\left(t, t_{w}\right)=\mathcal{G}\left(r=0 ; t, t_{w}\right)$. Generalizing the crossover approach (7) to this two-time quantity, one would expect the form

$$
C\left(t, t_{w} ; \epsilon\right)=\widehat{C}\left[\frac{L(t)}{L\left(t_{w}\right)}, \frac{\lambda(\epsilon)}{L\left(t_{w}\right)}\right],
$$

where $\widehat{C}$ is a scaling function. In the context of disordered ferromagnetic systems a superuniversality conjecture was proposed according to which the effect of disorder is simply accounted for by the slower growth of $L(t)$, while scaling functions entering correlation functions remains unchanged with respect to the clean case. This amounts to say that, e.g. for the autocorrelation function, $\widehat{C}(x, y)$ should not depend on the second entry. The superuniversality property was checked in several models, arriving at different conclusions. Indeed, while $d=1$ results [9, 10 clearly demonstrate the absence of superuniversality, in the cases with $d \geq 2$, there is evidence both in favor [17, 22] and against [6, 12] its validity, and there is presently an intense debate on the subject.

The original formulation of superuniversality was conceived for systems where inhomogeneities are introduced by disorder. The problem of determining the relevance of inhomogeneities on the scaling functions, however, may be posed on more general grounds and the simple systems studied in this paper may help the clarification of this issue. 


\section{COARSENING ON FRACTAL STRUCTURES}

We will consider in the following the Ising model defined by the Hamiltonian

$$
H[\sigma]=-J \sum_{\langle i j\rangle} \sigma_{i} \sigma_{j},
$$

where $\sigma_{i}= \pm 1$ is a unitary spin and $<i j>$ are nearest neighbors on a graph. The dynamics is introduced by randomly choosing a single spin and updating it with a transition rate that in the numerical simulations will be chosen in the Metropolis form

$$
w\left([\sigma] \rightarrow\left[\sigma^{\prime}\right]\right)=\min \left\{1, \exp \left[-\Delta E /\left(k_{B} T\right)\right]\right\} .
$$

Here $[\sigma]$ and $\left[\sigma^{\prime}\right]$ are the spin configurations before and after the move, and $\Delta E=H\left[\sigma^{\prime}\right]-H[\sigma]$. Phase-ordering is observed after a quench from an high initial temperature (assumed to be infinite in the following) to $T=0$ or to a temperature below the critical one $T_{c}$. In order to infer the properties and the physical content of the quantities introduced in the previous section, let us focus on the structures of the SG, of the TF, and of the SC [23, 24], representing prototypical examples of finitely ramified (the first two) and non-finitely ramified fractals (the latter). Indeed a wide class of fractal structures feature topological properties similar to the SG, TF and SC [25]. In finitely ramified fractals, an arbitrary large part of the structure can be disconnected by removing a finite number of cutting bonds. In general, they admit an ordered phase only at $T=0$. In a broad sense these structures can be considered a non trivial topological analogous of a low dimensional homogeneous systems with $d \leq d_{\ell}, d_{\ell}$ being the lower critical dimension. Non-finitely ramified graphs, on the other hand, can possess a low temperature ordered phase below a critical temperature $T_{c}$. All these fractals can be build starting from an elementary object, denoted as the first generation $G_{1}$, obtaining then an object of generation $G_{2}$ by combining $G_{1}$ parts, and then proceeding recursively as sketched in Fig. 1. In doing that, the linear size $L_{n}$ of the structure at generation $n$ grows as $L_{n}=f \cdot L_{n-1}$, where $f$ is constant which depends on the structure considered and, specifically, $f=2$ for the SG and the TF, and $f=3$ for the SC.

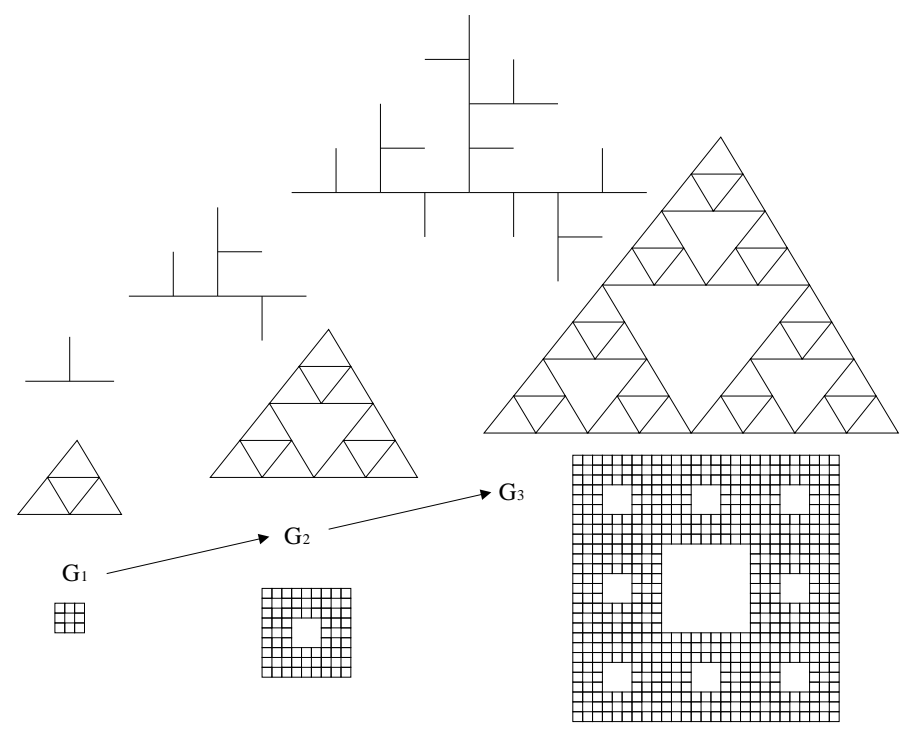

FIG. 1: Construction of the first 3 generations of the TF (upper part), SG (central part) and of the SC (lower part).

Since the Ising model on finitely ramified fractals is characterized by $T_{c}=0$, phase-ordering could in principle be truly asymptotic only after a quench to $T=0$, where however the dynamics is frozen because activated moves are prevented. Nevertheless, as explained in [19], a preasymptotic coarsening stage of diverging duration is observed for sufficiently low temperatures, similarly to what happens for instance on the $1 d$ lattice. We will focus on this stage of the dynamics to study coarsening on finitely ramified structures.

During the evolution, energetic barriers arise due to the mechanism sketched in Fig. 2 Let us consider the SG first, represented in the upper panel. The figure shows schematically the evolution of an interface which progressively 
spans a part of the structure. Initially, the position of the domain wall is outside the structure represented in the figure, in the left corner. This means that all the spins are, say, down. As time goes on, the interface enters the graph by moving across the intermediate position $I_{n}^{(\max )}$ indicated by a dotted green line (this means that spins on the left of the green line have been reversed up). The index $n$ refers to the fact that the interface is currently spanning the $n$-th generation of the fractal. When the spins of the whole generation $n$ have been reversed, the interface, depicted with two green arches, is located in the configuration $I_{n}^{(m i n)}$ on the four cutting bonds. Since the energy of a domain wall is $J$ times its length, it is clear that in $I_{n}^{(m i n)}$ the interface has a minimum energy $E_{n}^{(m i n)}$. On a finitely ramified structure, since the number of cutting bonds is finite this quantity is bounded from above and for large $n$, it becomes $n$-independent, i.e. $E_{n}^{(m i n)}=E^{(m i n)}$. For the $\mathrm{SG}, E^{(m i n)}=4 J$ for any $n$, since there are four cutting bonds for the interface, independently of $n$. Let us assume that the highest energy $E_{n}^{(\max )}$ of the system during the above process was reached in the (generic) configuration $I_{n}^{(\max )}$, so that a barrier of height $\mathcal{E}_{n}=E_{n}^{(\max )}-E_{n}^{(\min )}$ has been crossed. Now the interface must proceed again to the right in order to reverse all the spins of the next generation $n+1$, thus reaching the position $I_{n+1}^{(m i n)}$ located on the other four cutting bonds, and indicated by the couple of magenta arches (all the spins in the figure at this stage have then been reversed). Also this configuration has an energy equal to $E^{(\min )}$. The topology of the finitely ramified graph allows the system to attain this configuration by sequentially reversing parts of generation $n$ of the structure. For instance, in the Sierpinski gasket, by first reversing another triangle of generation $n$, say the lower-right one in Fig. 2. This event is analogous to the one described before. In particular, the interface in the intermediate position $I_{n+1}^{(\max )}$, depicted with a dashed-magenta line, is analogous to the previous one at $I_{n}^{(\max )}$ (dotted-green), except for the presence of an extra part around certain cutting bonds which, in the present example, are indicated with a dashed-magenta arch. For low temperatures the number of these cutting bonds tends to be minimized and it does not depend on the generation. Denoting with $E_{n+1}^{(\max )}$ the maximum energy reached by the system in the reversal of the $n+1$ generation, one concludes that $E_{n+1}^{(\max )}=E_{n}^{(\max )}+E_{c}$, where $E_{c}$ is the amount of extra energy due to the new part of interface (the dotted-magenta arch in the figure). Writing $E_{c}=J n_{c}$ where $n_{c}$ is the double of the number of broken bonds associated to the energy $E_{c}$, for the SG one has $n_{c}=4$. We recall that in general $n_{c}$ does not depend on $n$. Then

$$
\mathcal{E}_{n+1} \simeq \mathcal{E}_{n}+J n_{c}
$$

or, equivalently, rewriting $\mathcal{E}$ in terms of the size $L_{n}$ of the $n$-th generation

$$
\mathcal{E}\left(f \cdot L_{n}\right) \simeq \mathcal{E}\left(L_{n}\right)+J n_{c} .
$$

From this relation, dropping the index $n$, one has

$$
\mathcal{E} \simeq \frac{J n_{c}}{\ln f} \ln L
$$

Inserting this form into the Arrhenius relation (3) we arrive at a algebraic growth law as in Eq. (5) with

$$
\zeta \simeq a \frac{J}{T}
$$

and

$$
a \simeq \frac{n_{c}}{k_{B} \ln f}
$$

at low temperatures. The argument has been developed referring to the deterministic SG but it is expected to hold also for the TF (with $n_{c}=2$ ) and in general for finitely ramified deterministic and disordered structures, as for all of them an arbitrary large part can be disconnected by cutting a finite number of links.

A similar argument applies to non-finitely ramified structures, and it will be schematically illustrated for the SC with the help of the lower panel of Fig. 2 As for the SG, an interface enters the portion of the fractal considered from (say) the lower-left edge, crossing a region of generation $n$, passing through an high energy position depicted as an arch-shaped dashed green line. The evolution then proceeds towards the position $I_{n}^{(\min )}$ which in the figure is depicted by a continuous green line that roughly spans the diagonal of the $\mathrm{n}$-th generation. Here the energy reaches a minimum $E_{n}^{(\min )}$, because the largest hole available at generation $n$ is crossed, thus minimizing the number of disaligned spins. Then the domain wall moves to the next high energy configuration, depicted as a couple of dashed arch-shaped blue lines, and then to the minimum energy position $I_{n+1}^{(\min )}$ depicted with a continuous diagonal blue line. Eventually the 
configurations magenta are progressively reached by the interface. Notice that these configurations have the same energy of the blue ones. It is clear from the figure that the energies of the blue (or magenta) configurations occupied at generation $n+1$ are the double of those of the green ones at generation $n$. Hence one can write, in place of (14)

$$
\mathcal{E}\left(f \cdot L_{n}\right)=u \mathcal{E}\left(L_{n}\right),
$$

where $u=2$ for the SC. Hence, in place of Eqs. (15) we find

$$
\mathcal{E}\left(L_{n}\right) \simeq b J L_{n}^{d_{f l}},
$$

where $d_{f l}=\ln u / \ln f$ is in general the fractal dimension of the intersection of the fractal structure with a line, that is the border of the interface, corresponding to the cut set (e.g. it is $d_{f l}=\ln 2 / \ln 3$ for the $\mathrm{SC}$ considered in the figure). and from Eq. (3) we arrive at Eq. (2), with

$$
\psi=d_{f l} .
$$

This argument, developed for the SC, is expected again to be of general validity for all non-finitely ramified structures.

In conclusion, according to the above discussion, finitely (SG and TF) and infinitely (SC) ramified fractals represent the typical examples where barriers increase logarithmically and algebraically with $L$, Eqs. (4) 6) respectively, due to their topological properties. Correspondingly the growth law is expected to be a power law (with a temperature dependent exponent) or a logarithmic behavior, Eqs. (512) respectively. These behaviors should be observed when the energy scale $k_{B} T$ associated to temperature fluctuations is small with respect to the height $\mathcal{E}$ of the barriers. Conversely, for $\mathcal{E} \ll k_{B} T$, the effect of the barriers is negligible. Interfaces diffuse freely in this case and, in analogy to what observed on regular lattices, one expects to observe a power law behavior as in Eq. (9), characterized by the temperature independent exponent which describes the displacement $\left\langle x^{2}\right\rangle \propto t^{1 / z}$ of a random walker on the structure. For a fractal graph having spectral and fractal dimension $d_{s}, d_{f}$ respectively, it is $z=2 d_{f} / d_{s}$ [26]. We remark that this same power-law is predicted by approximate theories [27] to be the correct asymptotic growth-law, whereas our arguments indicate that it can only be preasymptotic.

All these behaviors can be fitted into the crossover scenario described by Eq. (77) provided that $z=d_{s} /\left(2 d_{f}\right)$ and

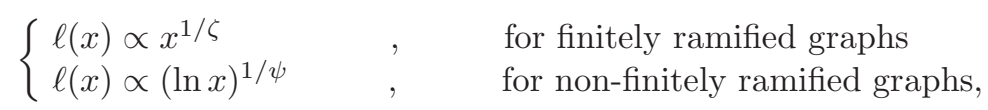

with $\zeta$ and $\psi$ given in Eqs. (16]20).

The crossover length $\lambda$ is given by the condition

$$
\mathcal{E}(\lambda) \simeq k_{B} T .
$$

Using Eqs. (15) and (19) this implies

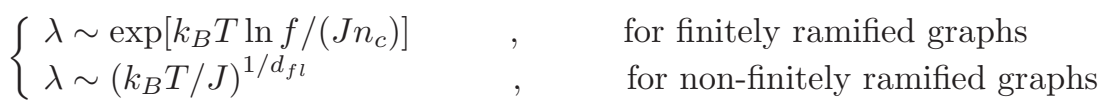

\section{NUMERICAL SIMULATIONS}

In this section we present the results of numerical simulations of the dynamics of the Ising model after a quench on the SG, on the TF, and on the SC. In the simulations we will consider systems with $n=9, n=8$ and $n=6$ generations respectively. With these choices finite size effects are not observed in the range of times considered. The system is prepared in a completely disordered state where spins are set to $\sigma_{i}= \pm 1$ randomly and independently on each site $i$, corresponding to an infinite temperature equilibrium initial state. The evolution is then implemented flipping randomly chosen single spins with the Metropolis rate (12) where $T$ is the temperature at its quench value. We always set $k_{B}=1$ and $J=1$ or, stated differently, we measure temperature in units of $J / k_{B}$. We consider a modified dynamics called no-bulk-flip, where spins which are aligned with all the nearest neighborg, namely in the bulk of an ordered domain, cannot flip. It was shown that this dynamics, which has been tested and used in a number of studies [19, 28], does not change the large-scale properties of the system and improves the speed of the simulation and the quality of the results. In order to grasp at least the basic physics inspiring the no-bulk-flip rule let us recall that in a coarsening system all the large-scale/long-time properties are uniquely determined by the motion of the ordered domains walls. Well inside the domains, regions of spins correlated over a length $\xi(T)$ can rapidly change sign 
over a characteristic time $\xi(T)^{z}$, where $z$ is the dynamical exponent. However, since for large times $t$ and $T<T_{c}, \xi(T)$ is always negligible with respect to $L(t)$, the flipping of these thermal islands only affect small-distances/short-times properties which are not the universal ones we are interested in. Further details on this accelerated dynamics can be found in [19, 28]. For the SG, we study quenches to different final temperatures in the range $T \in[0.8-3.4]$. For $T>3.4$ the system very rapidly reaches the disordered equilibrium state and the coarsening stage cannot be observed. On the other hand, for $T<0.8$ the dynamics becomes exceedingly slow to obtain reliable results with our computational resources. Similarly, for the TF we consider quenches in range of final temperatures $T \in[0.2-3]$ and for the SC $T \in[1-3]$.

On a homogeneous structure, assuming scaling (1), the equal time correlation function

$$
G(r ; t)=\left\langle\sigma_{i}(t) \sigma_{j}(t)\right\rangle,
$$

depends only on the distance $r$ between $i$ and $j$. From this the typical length $L(t)$ is usually extracted e.g. as the half height width $G(r=L(t) ; t)=1 / 2$. Although on a generic graph the notion of distance is not straightforward, one can reduce its definition to the usual one along certain directions, as done in [19, 20]. Proceeding analogously here we arrive at a determination of $L(t)$. This quantity is shown in Fig. 3 for quenches to different final temperatures.

A first trivial observation is that in the structures considered in the simulations there is a lower cutoff $L_{h o l e}$ on the size of the holes. Hence for $L(t)<L_{\text {hole }}$ the fractal nature of the graph is not revealed and we expect to observe the same behavior as on a homogeneous lattice. $L_{\text {hole }}$ is of the order of the size of the first generation and, in the cases considered here, $L_{\text {hole }} \simeq 3$. Very early data with $L(t)<L_{\text {hole }}$, therefore, do not describe the effect of the fractal structure and we will only refer the discussion to $L(t)>L_{\text {hole }}$.

For all structures considered here one sees very clearly a crossover from an early regime $L(t) \ll \lambda$, where curves for different temperatures roughly collapse, to a late stage with a strongly temperature dependent behavior. This is what one would expect from the picture described in the previous sections. According to the discussion of Sec. III, if such a crossover is described by Eq. (77), a number of predictions can be done, that we will check in the following.

\section{Crossover length:}

We start from the behavior of the crossover length $\lambda$ given in Eq. 23).

For the SG, using $f=2$ and $n_{c}=4$ one has $\ln f /\left(J n_{c}\right) \simeq 0.17$. Using Eq. (23) one concludes that, in the range of temperatures explored $(T \in[0.8-3.4]), \lambda$ can be varied at most by a factor 1.57. In Fig. 3 (upper left panel) we have indicated with a dashed horizontal line the value of $\lambda$ obtained from Eq. (23) by adjusting the proportionality constant in such a way that, for $L(t)>\lambda$ only the asymptotic power-law growth (21) (with $\zeta \neq z$ ) is observed. This can be done rather precisely for the lowest temperatures. Since $\lambda$ can be varied only by the small factor 1.57 it is clear that, even for the highest temperature one cannot obtain a significative range $L_{\text {hole }}<L(t)<\lambda$ to clearly determine the preasymptotic stage, where Eq. (9) should hold with a temperature-independent exponent $z=2 d_{f} / d_{s}$ (this law is represented by the dashed magenta line). Notice that at the highest temperatures $L(t)$ grows approximatively as in Eq. (9) but, as will be discussed below, for the different reason that the asymptotic temperature-dependent exponent $\zeta$ approaches the value $z=2 d_{f} / d_{s}$ at high temperatures. A similar situation is found for the TF (upper right panel). Here the smaller value of $n_{c}\left(n_{c}=2\right)$ allows to vary $\lambda$ by slightly larger factor i.e. 2.46. On this structure, then, at variance with the $\mathrm{SG}$ one should be able to detect the preasymptotic regime $L_{\text {hole }}<L(t)<\lambda$ at least for the larger temperatures.

For the SC we have plotted in Fig. 3 (second and third panel) the crossover length $\lambda$ obtained from Eq. (23), by using $d_{f l}=\ln 2 / \ln 3$ and adjusting the proportionality constant in such a way that the crossover from early powerlaw to asymptotic logarithmic behavior occurs around $\lambda$. As shown in Fig. 3 our estimate of $\lambda$ fit quite nicely at a semi-quantitative level with the data.

\section{Early stage:}

Next, let us consider the first regime, for $L(t) \ll \lambda$, that should obey the power-law behavior (9) with the temperature independent exponent $z=2 d_{f} / d_{s}$. As already discussed above, this preasymptotic regime is too short to be studied in the SG. For the TF this should in principle be observed at least at the largest temperature $T=2.5$ However, as it can be seen in Fig. 3 the crossover appears to be very broad, preventing a clearcut evidence on the preasymptotic behavior also on this structure.

On the contrary, for the SC at the highest quench temperature $T=3$ this stage lasts for more than a decade. Here one observes the expected power-law with exponent $z=2 d_{f} / d_{s}$ (such law is represented with the dashed magenta line). This shows that, indeed, in a preasymptotic regime barriers do not play any relevant role and interfaces perform a random walk on the graph. Notice also that, as already observed in [19, 20], an oscillatory behavior is superimposed on a globally increasing trend. This feature is observed in all the dynamical regimes and also in the SG and TF. 
Although the very limited extent of the time that can be reached in simulations does not allow to observe more than at most 1-2 oscillations, thus preventing any precise analysis, a semi-quantitative inspection of the data clearly suggests that these might be log-time periodic. This periodicity is observed in a number of apparently different phenomena, ranging from fracturing of heterogeneous solids [29], to stock market indexes [30], from magnetic systems with lack of translational symmetry [31], to phase separating fluids under shear [32]. This feature is generally associated to the presence of a discrete scale invariance [33] which in the present case induces a recurrent trapping of the interfaces when a complete generation of the fractal has been ordered. The presence of such log-periodic oscilations has been analytically proven for a random walker diffusing on these fractal structures [34].

\section{Late stage:}

The next step is the determination of the asymptotic growth law, namely the function $\ell$, which should behave as in Eq. (21).

For the SG and the TF it is clear that the curves oscillate around a net power-law behavior, as already observed in [19]. This is what was expected for finitely ramified graphs, according to Eq. (21). Our arguments provide the prediction (16]17) for the low-temperature behavior of $\zeta$. In order to check this we have plotted $1 / \zeta$ against temperature in the inset of the upper panels of Fig. 3, For low $\mathrm{T}$ the data show a good agreement with the expected behavior, which is represented by the dashed-blue lines. Notice that, in this case, there are no fitting parameters since also the value of the constant $a$ has been inferred.

In the case of the $\mathrm{SC}$, the logarithmic growth of $L(t)$ forces one to reach much longer times, particularly at low temperatures, and this in turn increases the computational effort limiting the possibility of a large statistics over many realizations of the thermal histories. Moreover the screening due to the oscillations is more severe. Nevertheless the data of the second panel of Fig 3 show quite unambiguously a crossover to a slower growth law around $L(t) \simeq \lambda$. According to our general picture this should be described by the logarithmic form of Eq. (21). In order to check this we have plotted in the third panel the same data but with an extra logarithm on the time axis. In this plot the form of Eq. (21) is a straight line with slope $1 / \psi$. This is very well consistent with our data for $L(t)>\lambda$. The numerical results show that the exponent $\psi$ decreases when $T$ is increased. In the limit of low temperature the value of Eq. (20) is predicted. The corresponding law is represented with a dashed-magenta line in the third panel of Fig. 3. Here one observes a very nice agreement with the data for the lowest temperature $(T=1)$, suggesting the correctness of our argument.

\section{Autocorrelation function:}

Finally, our simulations allows to comment on the role of the inhomogeneities on the scaling functions of correlation functions. We will consider in the following the autocorrelation function

$$
C\left(t, t_{w}\right)=\frac{1}{N} \sum_{i=1}^{N}\left\langle\sigma_{i}(t) \sigma_{i}\left(t_{w}\right)\right\rangle,
$$

where $N$ is the number of spins in the structure, for which the scaling form (10) is expected.

As discussed previously, because of the smallness of $\lambda$ on the SG and of the broad form of the scaling functions in the TF, the whole crossover pattern can be better detected in the SC. Hence we concentrate on this structure in the following. In Fig. 4 we plot the autocorrelation function measured after different waiting times $t_{w}$. The upper panel refers to the quench to the highest temperature $T=3$, where, according to the data of Fig. 3, one can access both the preasymptotic regime and the crossover to the late stage, and the role of the second entry of the scaling function in Eq. (10) can be studied. Interestingly, the crossover phenomenon is fully displayed in the figure. Indeed, the two curves for the smallest waiting times $\left(t_{w}=10\right.$ and $\left.t_{w}=20\right)$ almost collapse. The small residual dependence on $t_{w}$, namely the fact that the collapse is not perfect, is probably due to the fact that the presence of the crossover is already slightly felt even at these early times (but we cannot reduce $t_{w}$ further because of the constraint $L\left(t_{w}\right)>L_{h o l e}$ ). However, the picture shows that the separation between these two curves is much smaller than the one among the following ones, and this clearly indicates a convergence of the data to a limiting mastercurve for $L\left(t_{w}\right) \ll \lambda$. In view of Eq. (10) this means that the second entry $\lambda / L\left(t_{w}\right)$ is so large that $\widehat{C}\left[L(t) / L\left(t_{w}\right), \lambda(T) / L\left(t_{w}\right)\right] \simeq \widehat{C}\left[L(t) / L\left(t_{w}\right), \infty\right]$. Then, upon increasing $L\left(t_{w}\right)$, the second argument of $\widehat{C}$ decreases and becomes relevant. Indeed the collapse is lost and there is a clear tendency of the curves to move to larger values, as already noticed in 19]. This signals the crossover from the early stage to the late regime. Finally, for larger values of $L\left(t_{w}\right)\left(L\left(t_{w}\right) \gg \lambda\right)$ one has again a tendency to collapse on a mastercurve which corresponds to $\widehat{C}\left[L(t) / L\left(t_{w}\right), 0\right]$. This is very well verified for $t_{w}=1000,2000,4000$.

In order to complete the analysis we consider also the quench to the lower temperature $T=1$. In this case, since $\lambda$ is smaller the limiting curve $\widehat{C}\left[L(t) / L\left(t_{w}\right), 0\right]$ should be achieved at earlier $t_{w}$. Our results are plotted in the second 
panel of Fig. 4 At this low temperature the autocorrelation function is strongly oscillating. This is the counterpart of the periodic modulations already observed in the growth-law. These oscillations hinder somehow the collapse of the curves. Nevertheless, one observes that already from such small waiting times as $t_{w}=100$ onwards the curves do not show any tendency to move upwards, at variance with the case with $T=3$. This can be interpreted as due to the fact that, since $\lambda$ is much smaller at $T=1$, the collapse on the mastercurve $\widehat{C}\left[L(t) / L\left(t_{w}\right), 0\right]$ is already achieved at these early times. Clearly, since $\lambda$ is small, the pre-crossover collapse on $\widehat{C}\left[L(t) / L\left(t_{w}\right), \infty\right]$ is not observed here. Notice that the two mastercurves $\widehat{C}\left[L(t) / L\left(t_{w}\right), \infty\right]$ and $\widehat{C}\left[L(t) / L\left(t_{w}\right), 0\right]$ in the first panel are very well separated, clearly indicating the relevance of the second entry in the scaling function of Eq. (10). The whole behavior of $C$, which is captured by the two-parameter scaling (10), show unambiguously the relevance of the inhomogeneities, entering through the length $\lambda$, in determining the shape of the scaling functions.

\section{CONCLUSIONS AND PERSPECTIVES}

In this paper we have studied the phase-ordering kinetic of a ferromagnetic system with a scalar order-parameter on fractal graphs. We have proposed a scaling approach, inspired to renormalization group ideas, where a crossover between distinct dynamical behaviors is induced by the presence of a length $\lambda$ introduced by the topological properties of the graph. The transition between the early and the asymptotic stage is observed when the typical size $L(t)$ of the growing ordered domains reaches the crossover length $\lambda$. In this general framework, two classes of inhomogeneous substrates can be defined according to the nature of the activated processes which set in during the evolution. Specifically, we argue that on finitely ramified graphs the free energy barriers encountered by domains walls grow logarithmically with $L(t)$ while they increase as a power-law on all the other structures. This produces different asymptotic growth laws (power-laws vs logarithmic) and different dependence of the crossover length $\lambda$ on the model parameters. We have tested these ideas by numerical simulations of the Ising model on two model structures where, due to their relative simplicity, one can exhibit explicit predictions for the behavior of $L(t)$ and of $\lambda$, which conform very well to the numerical data.

The models studied in this paper can be considered as simple prototypical systems to understand the more general and still open problem of phase-ordering in inhomogeneous systems. A natural question is then if (and how) the results of this Article can be extended to more general situations. For instance, one might wonder if a similar picture holds in systems where dilution is random instead of being, as in this paper, deterministic. Following the arguments developed in Secs. IIIII one realizes that neither the deterministic character, neither the fractal nature are really determinant. Instead, the fundamental ingredient is weather the position of minimum energy of interfaces contain a number $n_{c}$ of broken bonds which is independent on $L(t)$ or if such number scales with (some power of) $L(t)$. This in turn is related to fact that the corresponding graph does not sustain a ferromagnetic phase $\left(T_{c}=0\right)$ or it does $\left(T_{c}>0\right)$, respectively. Extending this argument to the case of random dilution we can predict an asymptotic logarithmic growth law as in Eq. (2) for the randomly diluted (bond or site) Ising model with a fraction of occupied sites (or bonds) $p>p_{c}$, where $p_{c}$ is the percolation threshold. Right at $p=p_{c}$, on the other hand, we expect a power-law growth as in Eq. (5). Notice that, at variance with the fractal models studied in this Article, for randomly diluted systems one can tune continuously the parameter $p$ which controls the closeness to the threshold case $p=p_{c}$. Studies are in progress in order to test these ideas. Finally, it would be interesting to understand if similar concepts can be extended to interpret different system, where the inhomogeneous character is not due to dilution but to other agents, such as random coupling constants or spatially varying external fields.

[1] A.J. Bray, Adv. Phys. 43, 357 (1994).

[2] For reviews, see: F. Corberi, L. F. Cugliandolo and H. Yoshino, Growing length scales in aging systems in: Dynamical heterogeneities in glasses, colloids, and granular media, edited by L. Berthier, G. Biroli, J.-P. Bouchaud, L. Cipeletti and W. van Saarloos (University Press, Oxford, 2010). J.P.Bouchaud, L.F.Cugliandolo, J.Kurchan and M.Mezard, Out of equilibrium dynamics in spin-glasses and other glassy systems in: Spin Glasses and Random Fields, edited by A.P.Young (World Scientific, Singapore, 1997); L.F.Cugliandolo, Dynamics of glassy systems, in: Slow Relaxation and Non-Equilibrium Dynamics in Condensed Matter, edited by J.L.Barrat, J.Dalibard, J.Kurchan and M.V.Feigelman, (Springer-Verlag, Heidelberg, 2002).

[3] S. Puri, Phase Transitions 77, 469 (2004).

[4] M. Henkel and M. Pleimling, Europhys. Lett. 76, 561 (2006).

[5] M. Henkel and M. Pleimling, Phys. Rev. B 78, 224419 (2008).

[6] E. Lippiello, A. Mukherjee, S. Puri and M. Zannetti, Europhys. Lett. 90, 46006 (2010).

[7] H. Park and M. Pleimling, Phys. Rev. B 82, 144406 (2010). 
[8] Z.W. Lai, G.F. Mazenko and O.T. Valls, Phys. Rev. B 37, 9481 (1988). K. Tafa, S. Puri and D. Kumar, Phys. Rev. E 63, 046115 (2001).

[9] E. Lippiello, A. Mukherjee, S. Puri and M. Zannetti, Europhys. Lett. 90, 46006 (2010).

[10] F. Corberi, A. de Candia, E. Lippiello and M. Zannetti, Phys. Rev. E 65, 046114 (2002).

[11] D.S. Fisher, and D.A. Huse, Phys. Rev. B 38, 373 (1988). L.F. Cugliandolo, Physica A 389, 4360 (2010).

[12] F. Corberi, E. Lippiello, A. Mukherjee, S. Puri, and M. Zannetti, Phys. Rev. E 85, 021141 (2012).

[13] R.Burioni, D.Cassi, Jour. Phys. A 38 R45 (2005).

[14] H. Furukawa, J. Phys. Soc. Japan 58216 (1989); Phys. Rev. B 40, 2341 (1989). M. Zannetti, in Kinetics of Phase Transitions, edited by S. Puri and V. Wadhawan, (CRC Press, Boca Raton 2009), p. 153.

[15] S. J. Cornell, K. Kaski, and R. B. Stinchcombe, Phys. Rev. B 44, 12263 (1991). F. Corberi, C. Castellano, E. Lippiello, and M. Zannetti, Phys. Rev. E 65, 066114 (2002).

[16] S. Puri and N. Parekh, J. Phys. A 26, 2777 (1993). E. Oguz, A. Chakrabarti, R. Toral and J.D. Gunton, Phys. Rev. B 42, 704 (1990). E. Oguz, J. Phys. A 27, 2985 (1994).

[17] M. Rao and A. Chakrabarti, Phys. Rev. Lett. 71, 3501 (1993). C. Aron, C. Chamon, L.F. Cugliandolo and M. Picco, J. Stat. Mech. P05016 (2008).

[18] F. Corberi, E. Lippiello, A. Mukherjee, S. Puri, and M. Zannetti, J. Stat. Mech. P03016 (2011).

[19] R. Burioni, D. Cassi, F. Corberi, A. Vezzani Phys. Rev. E 75, 011113 (2007).

[20] R. Burioni, F. Corberi, A. Vezzani J. Stat. Mech. P02040 (2009).

[21] R. Paul, S. Puri and H. Rieger, Europhys. Lett. 68, 881 (2004); Phys. Rev. E 71, 061109 (2005). M. Henkel and M. Pleimling, Europhys. Lett. 76, 561 (2006); Phys. Rev. B 78, 224419 (2008).

[22] R. Paul, S. Puri and H. Rieger, Europhys. Lett. 68, 881 (2004); Phys.Rev. E 71, 061109 (2005). S. Puri, D. Chowdhury and N. Parekh, J. Phys. A 24, L1087 (1991); S. Puri and N. Parekh, J. Phys. A 25, 4127 (1992); A.J. Bray and K. Humayun, J. Phys. A 24, L1185 (1991). A. Sicilia, J. J. Arenzon, A. J. Bray and L. F. Cugliandolo, Europhys. Lett. 82, 1001 (2008).

[23] Y. Gefen, A. Aharony, Y. Shapir and B. B. Mandelbrot, J. Phys. A 16, 435 (1983); Y. Gefen, A. Aharonyt, Y. Shapir and B. B. Mandelbrot, J. Phys. A 17, 1277 (1984).

[24] R. Burioni, D. Cassi, F. Corberi, and A. Vezzani Phys. Rev. Lett. 96, 235701 (2006).

[25] R. Rammal Phys. Rev. B 28, 4872 (1983); Y. Lin, B. Wu, and Z. Zhang Phys. Rev. E 82, 031140 (2010); P. Buonsante, R. Burioni, and A. Vezzani Phys. Rev. E 84, 021105 (2011).

[26] J.P. Bouchaud and A. Georges, Phys. Rep. 195127 (1990).

[27] U. Marini Bettolo Marconi, Phys.Rev. E 57, 1290 (1998).

[28] F. Corberi, E. Lippiello, and M. Zannetti, Phys. Rev. E 63, 061506 (2001); Eur. Phys. J. B 24 (2001), 359; Phys.Rev. E 68, 046131 (2003); Phys. Rev. E 72, 056103 (2005) Phys.Rev. E 78, 011109 (2008). E. Lippiello, F. Corberi, A. Sarracino, and M. Zannetti, Phys. Rev. E 78, 041120 (2008). F. Corberi, and L.F. Cugliandolo, J. Stat. Mech. P05010 (2009).

[29] R.C. Ball and R. Blumenfeld, Phys. Rev. Lett. 65, 1784 (1990). M. Sahimi and S. Arbabi, Phys. Rev. Lett. 77, 3689 (1996). See also D. Sornette, Phys. Rep. 297, 239 (1998).

[30] D. Sornette and A. Johansen, Quantitative Finance 1, 452 (2001); Eur. Phys. Journ. B 17, 319 (2000); Physica A 245, 411 (1997).

[31] R.F.S. Andrade, Phys. Rev. E 61, 7196 (2000).

[32] F. Corberi, G. Gonnella, and A. Lamura, Phys. Rev. E 62, 8064 (2000); Phys. Rev. E 61, 6621 (2000); Phys. Rev. Lett. 83, 4057 (1999); Phys. Rev. Lett. 81, 3852 (1998). A. Lamura, G. Gonnella, F. Corberi, Eur. Phys. J. B 24, 251 (2001).

[33] D. Sornette, Chaos, Fractals, Self-organization and Disorder: Concepts and Tools, Springer 2004.

[34] P. J. Grabner and W. Woess, Stochastic Proc. Appl. 69127 (1997). 

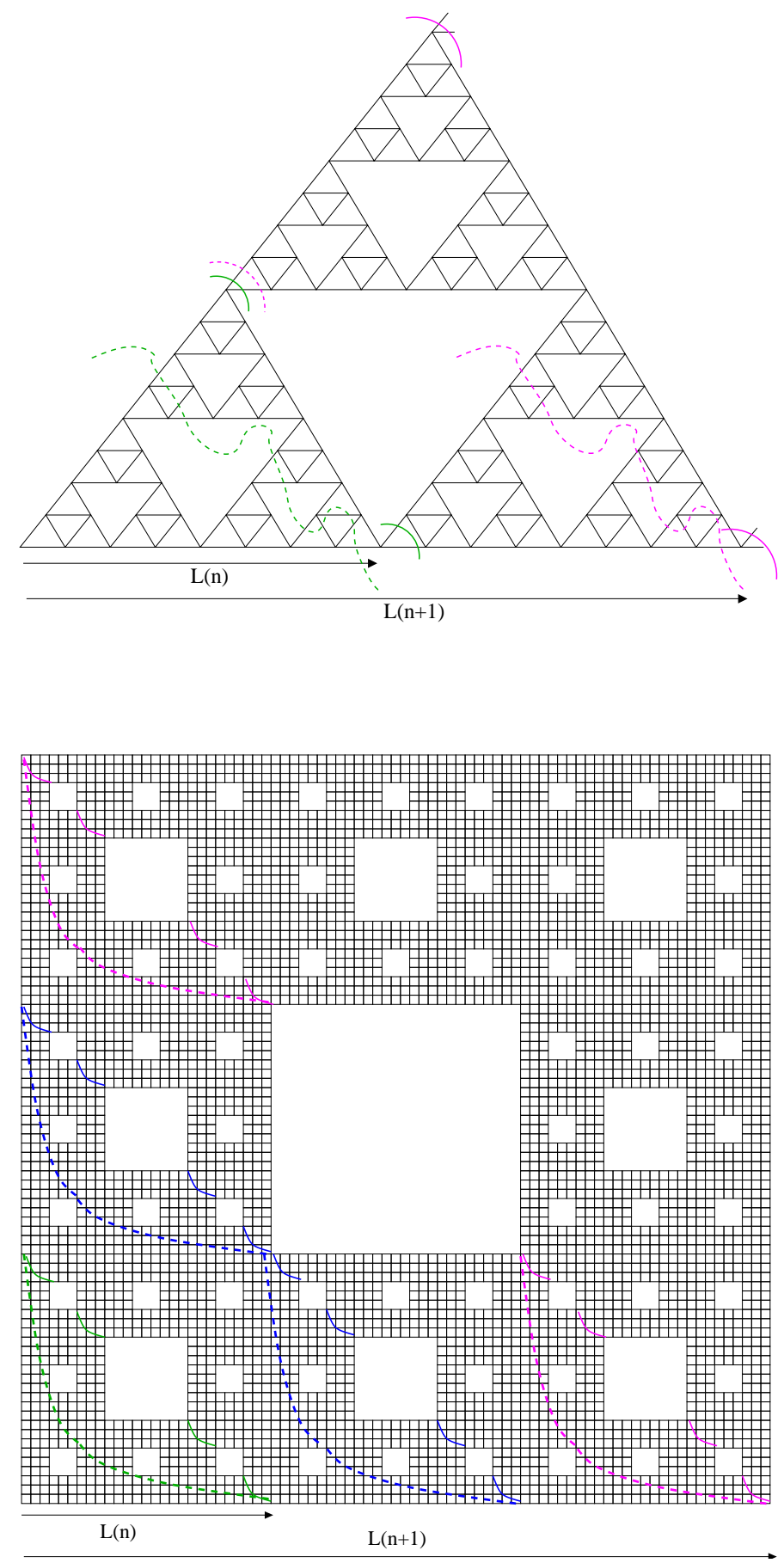

FIG. 2: Schematic description of energy barriers in the SG (upper panel) and in the SC (lower panel). For the SG, the green and the magenta arches represent the position of the interfaces in a configuration of minimum energy after a region of generation $n$ (and $n+1$ ) has been reversed. The dashed green and magenta lines represent the configuration of the interface with larger energy while reversing the structure of generation $n$ (and $n+1$ ). For the SC, the continuous green, blue, and magenta lines represent the position of minimum energy of an interface after the the reversing of a part of structure of generation $n$ (and $n+1)$. The corresponding dashed lines are the position of larger energy to be exceeded. 

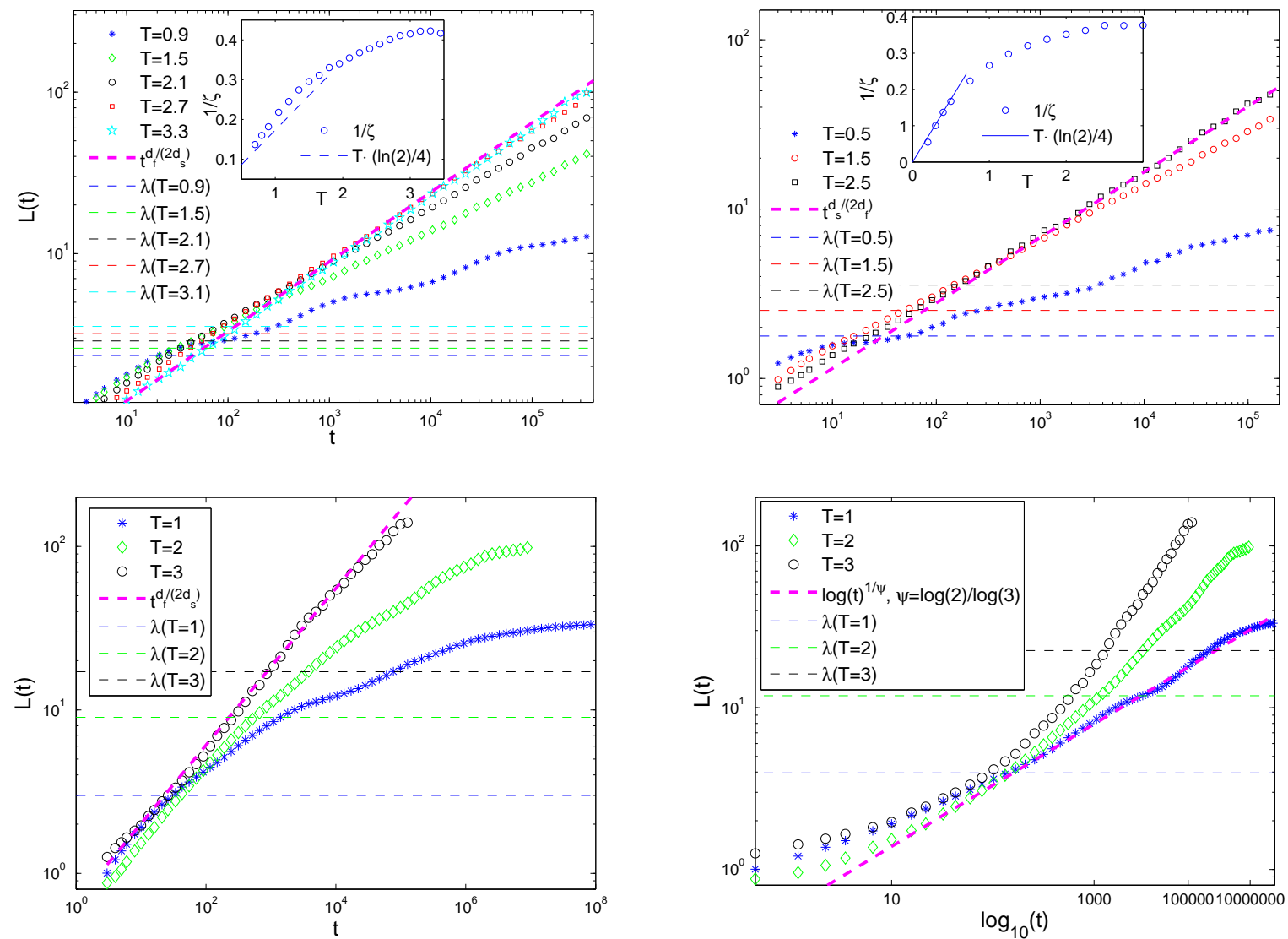

FIG. 3: The typical size $L(t)$ (symbols) is plotted against time in a log-log plot for an Ising model quenched to different final temperatures (see key) on the SG (upper left panel), the TF (upper right panel) and on the SC (lower panels). The bold dashed magenta line in the upper and lower left panels is the short time expected behavior $L(t) \sim t^{1 / z}$, with $z=2 d_{f} / d_{s}$, while in the lower right panel represents the asymptotic logarithmic law $L(t) \sim(\ln t)^{1 / \psi}$, with $\psi=d_{f l}=\ln 2 / \ln 3$. The horizontal dashed lines represent the crossover length $\lambda$. In the inset of the upper panels the exponent $1 / \zeta$, obtained from the data of the main part of the figure, is plotted against $T$. The dashed blue line is the prediction (16]17). 

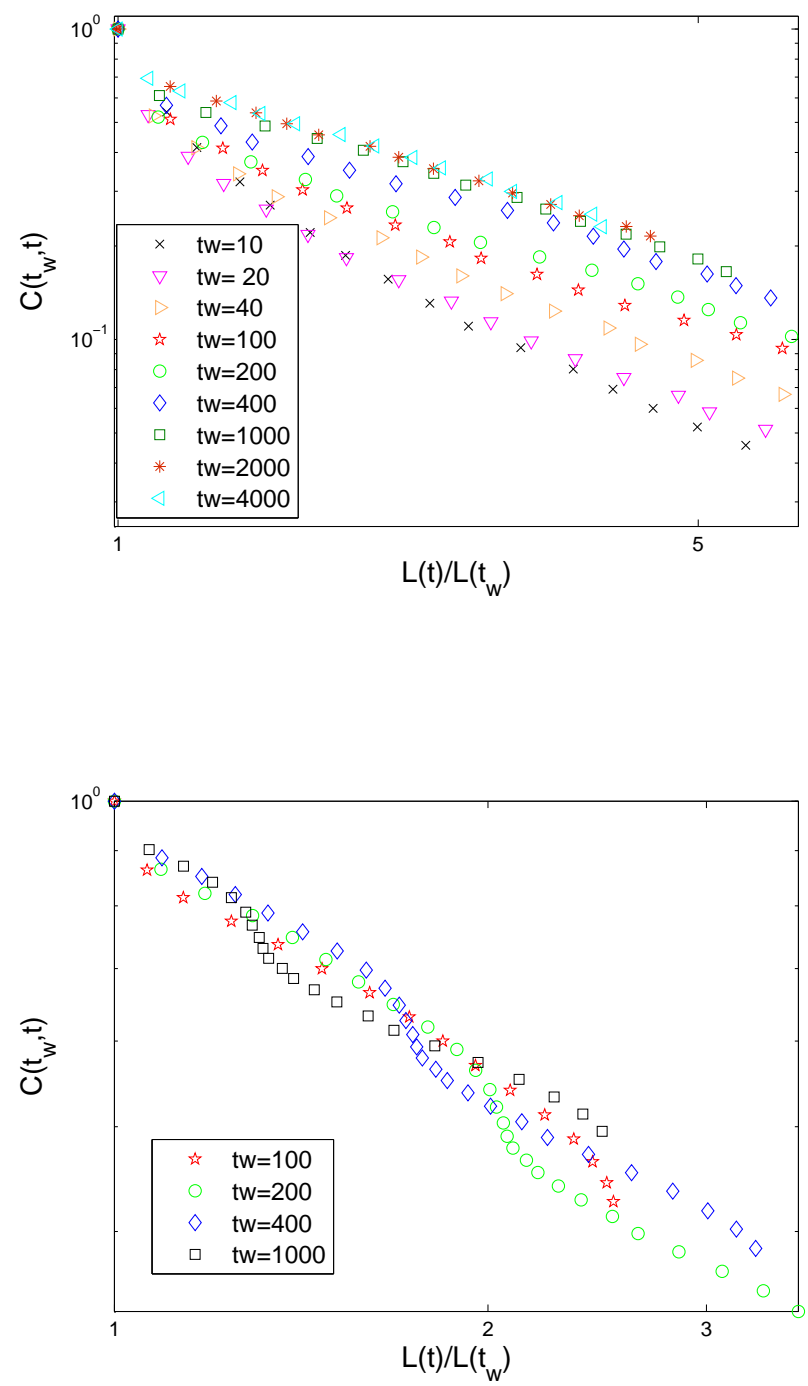

FIG. 4: The autocorrelation function $C\left(t, t_{w}\right)$ is plotted against $L(t) / L\left(t_{w}\right)$ for different values (see key) of $t_{w}$. In the first panel a quench to $T=3$ is considered, while in the second it is $T=1$. 